\title{
DOMESTICACIÓN Y FITOQUÍMICA DE QUASSIA AMARA (SIMAROUBACEAE) EN EL TRÓPICO HÚMEDO DE COSTA RICA
}

\author{
Roberto Díaz ${ }^{1}$, Lorena Hernández ${ }^{2}$, Rafael Ocampo $^{1} \&$ José F. Cicció*2 \\ ${ }^{1}$ Jardín Agroecológico Bougainvillea, S.A., Costa Rica. ${ }^{2}$ Centro de Investigaciones en Productos \\ Naturales (CIPRONA) y Escuela de Química, Universidad de Costa Rica, 2060 San José, Costa Rica. \\ *<jfciccio@equi.ucr.ac.cr>
}

\begin{abstract}
A description of some characteristics of the plant Quassia amara, including taxonomy, distribution, uses, pharmacognosy and some biological activities, is given. Experimental activities related to ecology, conservation, and domestication in Costa Rica are described. Details of the agro-ecological cultivation, importance of the phytochemical methods, and their applications to control the production of quassin and neoquassins according to the age of the plants are discussed.

RESUMEN. Se describen algunas características de Quassia amara, tales como taxonomía, distribución, usos, farmacognosia y algunas actividades biológicas. Se presentan actividades experimentales relacionadas con ecología, conservación y domesticación en Costa Rica. Se discuten detalles sobre el cultivo agroecológico, los métodos fitoquímicos y los resultados de su aplicación para controlar la producción de cuasina y neocuasinas, según la edad de las plantas.
\end{abstract}

Palabras Clave / Key words: Quassia amara, Simaroubaceae, domesticación de plantas / plant domestication, fitoquímica / phytochemistry, actividad biológica / biological activity.

\section{A. INTRODUCCIÓN}

En el Jardín Agroecológico Bougainvillea, ubicado en la zona atlántica de Costa Rica, América Central (altitud entre 43 y $\left.183 \mathrm{~m} ; 10^{\circ} 01^{\prime} 03.1^{\prime \prime} \mathrm{N}, 83^{\circ} 19^{\prime} 41.6^{\prime \prime} \mathrm{W}\right)$, se desarrolla un proceso de domesticación de Quassia amara L., con la finalidad de contribuir a la disminución de la recolección de poblaciones de plantas naturales del bosque y de promover una mayor conservación del ambiente, mediante prácticas de cultivo apropiadas, basadas en un modelo agroecológico. Además, se puede contar con materia prima de calidad, en forma de extractos estandarizados utilizados para la formulación de productos medicinales, saborizantes, productos veterinarios e insecticidas. De este modo, se asegurará un abastecimiento continuo de materia prima en un sistema sustentable.

\section{Taxonomía y distribución}

El género simaroubáceo Quassia fue publicado por Linneo en 1762 (Species Plantarum, Ed. secunda 1: 553. 1762). El nombre de esta planta, usada tradicionalmente contra la malaria, recuerda al esclavo curandero Quassi, quien descubrió en Surinam sus propiedades medicinales (Fernand 2003). La familia Simaroubaceae posee una distribución pantropical (América tropical, África, Asia, Malasia y noreste de Australia), pero su centro de distribución principal se halla en América tropical. Se divide en seis subfamilias con alrededor de 30 géneros y 150 a 200 especies arbustivas y arbóreas (Simão et al. 1991, Fernando \& Quinn 1995, Poveda 1995). Resulta difícil determinar la distribución natural original de Quassia amara, ya que esta especie se ha utilizado económicamente con varios propósitos durante mucho tiempo. No obstante, diversos autores la consideran nativa desde el sur de México y las Antillas hasta el norte de América del Sur (Cronquist 1944).

Esta planta, caracterizada por poseer constituyentes amargos, principalmente en corteza, madera y semillas, se ha confundido, en el mercado, con Picrasma excelsa (Sw.) Planch., que se conoce con el mismo nombre común quassia o cuasia. Esta especie contiene muchos de los constituyentes químicos de Q. amara y se utiliza básicamente para los mismos tratamientos medicinales. Los árboles de $P$. excelsa son más grandes (hasta unos $25 \mathrm{~m}$ ) que los de Q. amara y habitan en las Antillas, especialmente en Jamaica (Morton 1981), y también crecen en forma natural en Costa Rica, en las cordilleras de Guanacaste y de Tilarán, a altitudes que oscilan entre 1100 y $1500 \mathrm{~m}$ (Zamora et al. 2000).

\subsection{Distribución natural en Costa Rica}

Las zonas de vida donde se encuentra Quassia amara abarcan cerca del $50 \%$ del territorio de Costa Rica 
(Villalobos 1995a, Villalobos 1996). La mayor parte de las zonas donde habita corresponden a bosques húmedos o muy húmedos; un número de plantas mucho menor se halla en zonas bajas de bosque seco. La altitud máxima donde se ha observado es de $c a .350 \mathrm{~m}$, cerca del cerro La Cangreja de Puriscal; la mínima es de $3 \mathrm{~m}$ en la región costera del Pacífico sur. Es probable que la altitud y la temperatura limiten la distribución natural de esta especie.

\subsection{Breve descripción de la planta}

Es un arbusto o árbol pequeño de 3 a $6 \mathrm{~m}$ de altura. En Talamanca, Ling (1995) determinó promedios de $1,88 \mathrm{~m}$ de altura y $1,96 \mathrm{~cm}$ de diámetro a la altura del pecho (dap), con máximos de 6,5 m y 6,16 cm de dap. Las hojas son imparipinnadas, alternas, con el raquis alado, con 3 ó 5 foliolos sésiles, opuestos. Los foliolos son oblongos u obovados, de 5 a $11 \mathrm{~cm}$ de largo, glabros y membranáceos (Holdridge et al. 1997). Las flores son muy vistosas, en racimos, con 5 pétalos lanceolados de color rojo o anaranjado, de 2 a $5 \mathrm{~cm}$ de largo. Los frutos son drupas que al madurar se vuelven de color negro, de 1 a 1,5 cm de largo (Morton 1981).

\subsection{Nombres vernaculares}

1.3.1. En Costa Rica: cuasia, hombre grande, hombrón, quini, bakonki, kläklö, webblakló, pito kicha (Fournier \& García 1998).

1.3.2. En América Latina: Bitterhout, Bitter Wood, crucete, cuasia amarga, guabito amargo, guavo, kwasibita, kwassie bita, murupa, palo de cuasia, palo isidoro, pau amarelo, pau quassia, puesilde, quacia amarga, Quashi Bitters, Quassia, quassia amarga, quassia de Caiena, Quassia Surinam, quina cayenna, quinine de cayenne, Suriname wood (Morton 1981).

Alemán: Jamaika Quassienholz; francés: Bois de quassia de la Jamaïque; inglés: Quassia wood; Jamaica Quassia; italiano: Quassio, legno amaro; portugués: Lenho de quássia.

\section{Usos}

\subsection{Usos medicinales}

Pittier (1957) indica que esta planta era uno de los principales remedios de los indios de Costa Rica. Ellos dividían el tronco en trozos de 30 a $60 \mathrm{~cm}$, los llevaban en sus viajes y los utilizaban contra la fiebre. En la actualidad, en Talamanca es usada como planta medicinal por la población indígena, por los afrocaribeños de la costa y por los agricultores ladinos de otros sectores del cantón.

La infusión de la corteza y de la madera se ha usado como tónico amargo, estimulante del apetito, febrífugo $y$, en forma de enemas, antihelmíntico contra los oxiuros (Núñez-Meléndez 1975). Según García (1992), la cuasina amorfa o cristalina obtenida de la cuasia "es capaz de aumentar la secreción de las glándulas salivales, hígado y riñones, exitar las fibras musculares del tubo digestivo, aparato uropoyético y canal excretor de la bilis, aumentando la actividad de las mucosas y las secreciones normales". Según Segleau (2001) se toma en infusión contra las manchas de la cara, causadas por problemas hepáticos, y se usa como amebicida. La decocción de la madera se utiliza popularmente para el control de la diabetes mellitus (Holdridge \& Poveda 1975). En homeopatía se recomienda para debilidad, dispepsia, hepatosis e ictericia (Morton 1981). En Europa se preparan vinos amargos por maceración de una pequeña cantidad de madera, para combatir náuseas (Cecchini 1971).

La decocción de la planta se usa para lavar la cabeza, con el objeto de matar piojos (CENAP 1991).

\subsection{Indicaciones}

En la actualidad el leño de cuasia se usa como tónico amargo para estimular el apetito y como digestivo (Cañigueral et al. 1998).

\subsection{Contraindicaciones}

Las mujeres no deben ingerir cuasia durante la menstruación, porque produce cólicos uterinos (Manfred 1947). Altas dosis producen vómito. No es recomendada para niños. Puede producir una disminución de la presión arterial y no se debe ingerir acompañada de bebidas alcohólicas. Si se toma en exceso puede causar depresión (Segleau 2001).

\subsection{Efectos secundarios}

Si la dosis es elevada, se produce irritación de la mucosa gástrica y puede provocar el vómito. Por vía parenteral, la cuasina es tóxica: provoca disminución de la frecuencia cardíaca, temblores musculares y parálisis (Cañigueral et al. 1998).

\subsection{Otros usos}

En la industria alimentaria, los extractos de corteza y madera se emplean como amargo en tónicos digestivos, en bebidas carbonatadas tónicas y en licores (Morton 1981). También, se usan en vinos digestivos que combaten la pesadez de estómago, la digestión difícil y la flatulencia (Cecchini 1971). Desde el punto de vista agrícola, extractos de esta planta se usaron como insecticida en Estados Unidos de Norteamérica desde 1850 y en Europa desde 1880 (Morton 1981). Desde esa época, se han realizado numerosas investigaciones, en 
las que variados extractos han resultado efectivos contra diversos tipos de plagas de insectos, sobre todo áfidos y lepidópteros (Cáceres et al. 1995a).

\subsection{Regulaciones}

En Europa, la cuasina se permite con un límite máximo de $5 \mathrm{mg} / \mathrm{kg}$ en productos alimenticios y bebidas, con las excepciones de $10 \mathrm{mg} / \mathrm{kg}$ en confitería, en forma de pastillas, y $50 \mathrm{mg} / \mathrm{kg}$ en bebidas alcohólicas (European Commission. SCF/CS/FLAV/FLAVOUR/29 Final Opinion of the Scientific Committee on Food on quassin. 25 July 2002).

En los Estados Unidos de Norteamérica, "Quassia" (de Picrasma excelsa o Quassia amara) se considera de uso seguro en alimentos ("may be safely used in food"; CFR 2002. US Code of Federal Regulations. Title 21. § 172.510).

\section{Farmacognosia}

\subsection{Leño de cuasia (Quassiae lignum)}

La cuasia (U.S.P. 1820 a 1936; N.F. 1936 a 1960) es la madera de Quassia amara L. o de Picrasma excelsa (Sw.) Planch., conocidas comercialmente como cuasia de Surinam y cuasia de Jamaica, respectivamente (Claus \& Tyler 1965).

La droga consiste en virutas, astillas o aserrín de color amarillento. Si contiene corteza, también habrá partículas de color grisáceo. Tanto la droga como los extractos de la corteza y la madera son muy amargos y poseen escaso olor.

\subsection{Descripción microscópica}

La madera se reconoce al microscopio por los falsos anillos de crecimiento anual (bandas de parénquima); presenta radios medulares formados por una o dos filas de células de ancho y de 20 a 25 células de largo. Además, carece casi completamente de cristales de oxalato de calcio (Cañigueral et al. 1998).

\subsection{Composición}

La cuasina cruda, extraída de la madera de Quassia amara, contiene una serie de seco-triterpenoides denominados cuasinoides. Los más abundantes son cuasina, neocuasina, 18-hidroxicuasina y 14,15deshidrocuasina (Robins \& Rhodes 1984) (Fig. 1).

Se han aislado e identificado, en pequeñas cantidades, varios derivados de cuasina; entre ellos $16 \alpha-O-m e t i l n e o c u a s i n a, 1 \alpha-O-m e t i l c u a s i n a$, paraína, 11-acetilparaína, isoparaína y 12 $\alpha$-hidroxi-13,18deshidroparaína (Barbetti et al. 1993) (Fig. 2). De la savia se aisló simalikalactona D y cuasimarina, un cuasinoide que mostró actividad antileucémica (Kupchan \& Streelman 1976) (Fig. 3).

Otro grupo importante de compuestos hallados en cuasia son alcaloides indólicos de dos tipos:

El primer grupo corresponde al tipo de la cantina: 3-metilcantin-2,6-diona, 5-hidroxi-3-metil-4-metoxicantin-2,6-diona, 3-metilcantin-5,6-diona, 2-metoxicantin-6-ona, 3- $N$-óxido de 5-hidroxi-4-metoxicantin-6-ona y 5-hidroxi-4-metoxicantin-6-ona (Barbetti et al. 1993, Barbetti et al. 1987, Barbetti et al. 1990, Njar et al. 1993) (Fig. 4). El segundo grupo consta de derivados de la $\beta$-carbolina: 1 -vinil-4,8-dimetoxi- $\beta$ carbolina, 1-metoxicarbonil- $\beta$-carbolina y $N$-metoxi1 -vinil- $\beta$-carbolina (Barbetti et al. 1987, Furlan et al. 1994) (Fig. 5).
Cuasina

Neocuasina

18-hidroxicuasina

14,15-deshidrocuasina

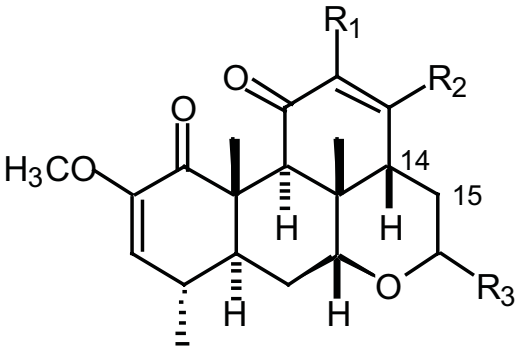

$\mathbf{R}_{1}$

$\mathrm{OCH}_{3}$

$\mathrm{OCH}_{3}$

$\mathrm{OCH}_{3}$

$\mathrm{OCH}_{3}$

\begin{abstract}
$\mathbf{R}_{2}$
\end{abstract}
$\mathrm{CH}_{3}$

$\mathrm{CH}_{3}$

$\mathrm{CH}_{2} \mathrm{OH}$

$\mathrm{CH}_{3}$
$\mathbf{R}_{3}$

$=\mathrm{O}$

$\mathrm{H} \mathrm{OH}$

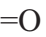

$=\mathrm{O}$

Fig. 1. Cuasinoides más comunes en Quassia amara. 


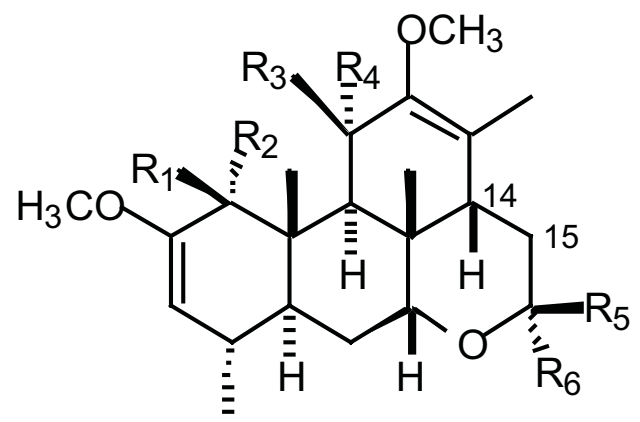

\begin{tabular}{|c|c|c|c|c|c|c|}
\hline & $\mathbf{R}_{1}$ & $\mathbf{R}_{2}$ & $\mathbf{R}_{3}$ & $\mathbf{R}_{4}$ & $\mathbf{R}_{5}$ & $\mathbf{R}_{6}$ \\
\hline 11- $\alpha-O-(\beta$-D-glucopiranosil)- & $\mathrm{O}$ & $\mathrm{O}$ & O-Glc & $\mathrm{H}$ & $\mathrm{H}$ & $\mathrm{OMe}$ \\
\hline 16- $\alpha-O$-metilneocuasina & $\mathrm{O}$ & $\mathrm{O}$ & $\mathrm{O}$ & $\mathrm{O}$ & $\mathrm{H}$ & $\mathrm{OMe}$ \\
\hline 1-dihidro- $\alpha$-metoxicuasina & $\mathrm{OMe}$ & $\mathrm{H}$ & $\mathrm{O}$ & $\mathrm{O}$ & $\mathrm{O}$ & $\mathrm{O}$ \\
\hline
\end{tabular}

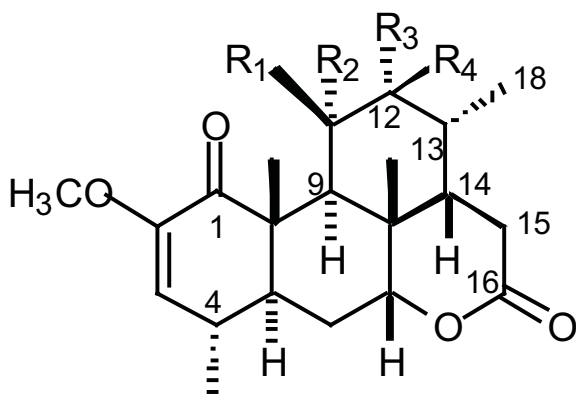

paraína

$\begin{array}{llll}\mathbf{R}_{\mathbf{1}} & \mathbf{R}_{\mathbf{2}} & \mathbf{R}_{\mathbf{3}} & \mathbf{R}_{\mathbf{4}} \\ \mathrm{H} & \mathrm{OH} & \mathrm{O} & \mathrm{O} \\ \mathrm{H} & \mathrm{OAc} & \mathrm{O} & \mathrm{O} \\ \mathrm{O} & \mathrm{O} & \mathrm{H} & \mathrm{OH} \\ \mathrm{H} & \mathrm{OH} & \mathrm{OH} & \mathrm{H}\end{array}$

Fig. 2. Cuasinoides minoritarios en Quassia amara. 


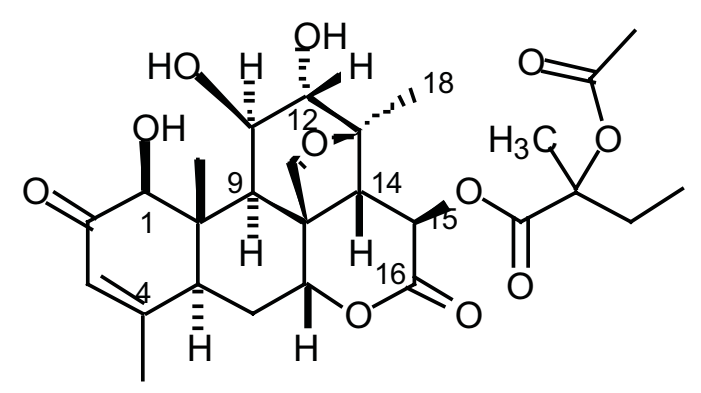

Cuasimarina

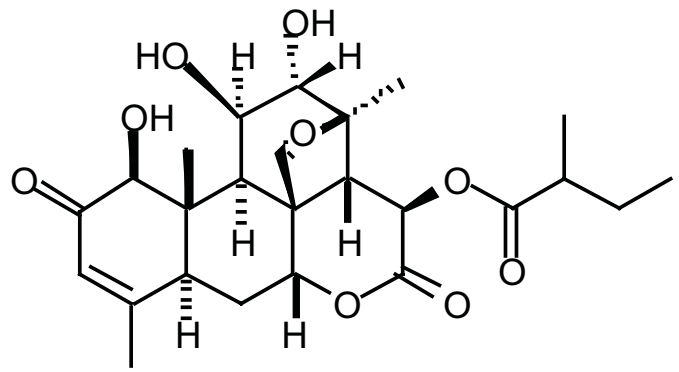

Simalikalactona D

Fig. 3. Cuasinoides aislados de la savia de Quassia amara.

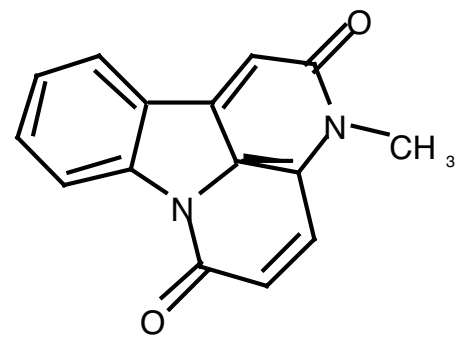

3-metilcantin-2,6-diona

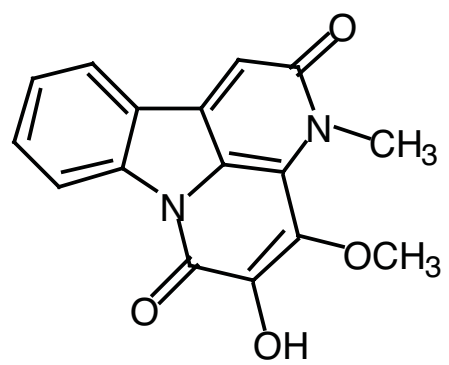

5-hidroxi-3-metil-4-metoxicantin-2,6-diona

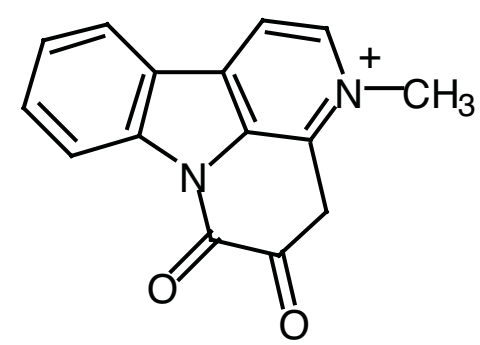

2-metoxicantin-6-ona

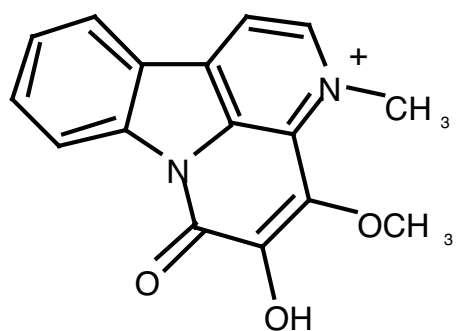

5-hidroxi-3-metil-4-metoxicantin-2,6-diona

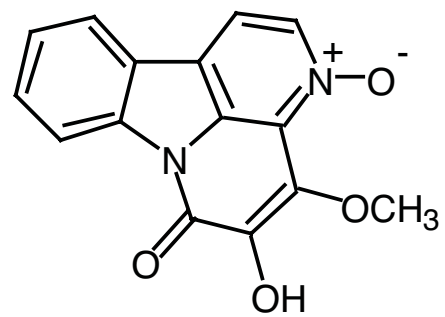

$3 N$-óxido de 5-hidroxi-4-metoxicantin-6-ona

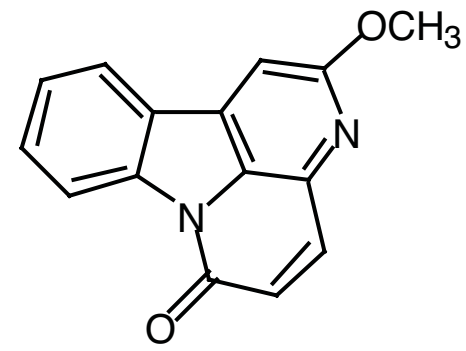

3-metilcantin-5,6-diona

Fig. 4. Alcaloides de la cantina hallados en Quassia amara. 


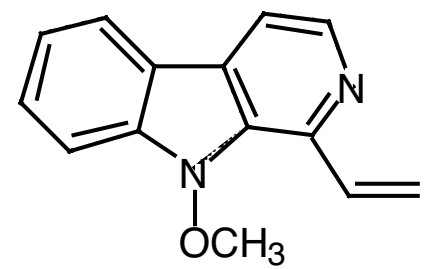

$N$-metoxi-1-vinil- $\beta$-carbolina

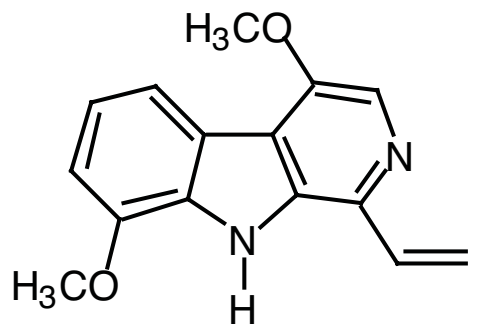

1-vinil-4,8-dimetoxi- $\beta$-carbolina<smiles>COC(=O)c1nccc2c1[nH]c1ccccc12</smiles>

1-metoxicarbonil- $\beta$-carbolina

Fig. 5. Alcaloides de la $\beta$-carbolina hallados en Quassia amara. 


\section{Actividad biológica experimental}

En el Cuadro 1 se enumeran algunas de las actividades biológicas observadas en extractos de Q. amara.

Cuadro 1. Lista de algunas actividades biológicas inducidas por extractos de Quassia amara.

\begin{tabular}{|c|c|c|c|c|c|}
\hline ACTIVIDAD POSITIVA & Organismo & $\begin{array}{l}\text { PARTE } \\
\text { USADA }\end{array}$ & EXtracto & $\begin{array}{l}\text { ORIGEN DE LA } \\
\text { PARTE USADA }\end{array}$ & REFERENCIA \\
\hline Antiviral & VIH & corteza & D.N.D. ${ }^{1}$ & Bolivia & $\begin{array}{l}\text { Abdel-Malek et } \\
\text { al. } 1996\end{array}$ \\
\hline Larvicida & $\begin{array}{l}\text { Culex } \\
\text { quinquefasciatus }\end{array}$ & hojas & EtOH 95\% & India & $\begin{array}{l}\text { Evans \& Raj } \\
1988\end{array}$ \\
\hline Antimalárica & Plasmodium berghei & hojas & $\mathrm{MeOH}$ & Nigeria & Ajaiyeoba 1999 \\
\hline Antitumoral & Leucemia P-388 & savia seca & savia seca & Costa Rica & $\begin{array}{l}\text { Kupchan \& } \\
\text { Streelman 1976, } \\
\text { Ferrigni et al. } \\
1982\end{array}$ \\
\hline Antitumoral & Leucemia P-388 & madera & $\mathrm{H}_{2} \mathrm{O}$ & Costa Rica & $\begin{array}{l}\text { Kupchan \& } \\
\text { Streelman } 1976\end{array}$ \\
\hline Insecticida & Áfidos & D.N.D. & $\mathrm{H}_{2} \mathrm{O}$ & Surinam & Roark 1947 \\
\hline Insecticida & $\begin{array}{l}\text { Pulgón verde: Mysus } \\
\text { persicae }\end{array}$ & madera & EtOH 95\% & Costa Rica & $\begin{array}{l}\text { Rodríguez \& } \\
\text { Blanco } 1995\end{array}$ \\
\hline Insecticida & $\begin{array}{l}\text { mosca blanca: Bemisia } \\
\text { tabaci }\end{array}$ & madera & $\begin{array}{l}\mathrm{H}_{2} \mathrm{O} \text { y } \\
\mathrm{MeOH}\end{array}$ & Costa Rica & $\begin{array}{l}\text { Cubillo et al. } \\
1997\end{array}$ \\
\hline $\begin{array}{l}\text { Antiparasitaria } \\
\text { (pediculicida) }\end{array}$ & $\begin{array}{l}\text { Pediculus humanus } \\
\text { humanus L. }\end{array}$ & madera & EtOH $75 \%$ & D.N.D. & Ninci 1991 \\
\hline Antimicrobiana & varias cepas & madera & EtOH $50 \%$ & Costa Rica & $\begin{array}{l}\text { Cáceres et al. } \\
1995 \mathrm{~b}\end{array}$ \\
\hline Protector gástrico & $\begin{array}{l}\text { Mus musculus y } \\
\text { Rattus norvegicus }\end{array}$ & corteza & $\mathrm{H}_{2} \mathrm{O}$ & Costa Rica & $\begin{array}{l}\text { Badilla et al. } \\
1998\end{array}$ \\
\hline $\begin{array}{l}\text { Tóxica aguda: } \\
\text { negativa }\end{array}$ & $\begin{array}{l}\text { Ratones machos NGP- } \\
\text { UCR (oral) }\end{array}$ & Madera & $\mathrm{H}_{2} \mathrm{O}$ & Costa Rica & $\begin{array}{l}\text { García et al. } \\
\text { 1996-1997 }\end{array}$ \\
\hline $\begin{array}{l}\text { Tóxica aguda: } \\
\text { positiva }\end{array}$ & $\begin{array}{l}\text { Ratones machos NGP- } \\
\text { UCR (intraperitoneal) }\end{array}$ & Madera & $\mathrm{H}_{2} \mathrm{O}$ & Costa Rica & $\begin{array}{l}\text { García et al. } \\
\text { 1996-1997 }\end{array}$ \\
\hline $\begin{array}{l}\text { Facilitadora del } \\
\text { tránsito intestinal }\end{array}$ & $\begin{array}{l}\text { Ratones machos NGP- } \\
\text { UCR }\end{array}$ & Madera & $\mathrm{H}_{2} \mathrm{O}$ & Costa Rica & $\begin{array}{l}\text { García et al. } \\
\text { 1996-1997 }\end{array}$ \\
\hline Antiulcerogénica & Ratones machos Swiss & $\begin{array}{l}\text { Corteza } \\
\text { seca }\end{array}$ & $\begin{array}{l}\text { EtOH } 100 \% \\
\text { EtOH } 70 \% \\
\mathrm{CH}_{2} \mathrm{Cl}_{2} \\
\text { Hexano }\end{array}$ & $\begin{array}{l}\text { República } \\
\text { Dominicana }\end{array}$ & $\begin{array}{l}\text { Toma et al. } \\
2002\end{array}$ \\
\hline $\begin{array}{l}\text { Antiedematogénica, } \\
\text { analgésica, sedante }\end{array}$ & Ratones machos Swiss & $\begin{array}{l}\text { Corteza } \\
\text { seca }\end{array}$ & $\begin{array}{l}\text { EtOH } 100 \% \\
\text { EtOH } 70 \% \\
\mathrm{CH}_{2} \mathrm{Cl}_{2} \\
\text { Hexano }\end{array}$ & $\begin{array}{l}\text { República } \\
\text { Dominicana }\end{array}$ & $\begin{array}{l}\text { Toma et al. } \\
2003\end{array}$ \\
\hline Esterilizante & Ratas albinas & corteza & $\mathrm{CHCl}_{3}$ & India & $\begin{array}{l}\text { Parveen et al. } \\
2003\end{array}$ \\
\hline
\end{tabular}

${ }^{1}$ D.N.D.: Datos no disponibles

5. Ecología, conservación y domesticación

\subsection{Ecología}

Los primeros trabajos realizados en Costa Rica fueron parte del Proyecto Conservación para el Desarrollo
Sostenible en América Central (Proyecto Olafo) del Centro Agronómico Tropical de Investigación y Enseñanza (CATIE), entre 1989 y 1997. Se trabajó en la Reserva Indígena Kéköldi, situada en la zona de Baja Talamanca (Ling 1995, Marmillod et al. 1995). Se preparó un mapa de las poblaciones silvestres de Quassia 
amara de esta zona; la de mayor tamaño se ubicó en la reserva Kéköldi, con una extensión aproximada de 100 hectáreas, con diferentes abundancias dependiendo del hábitat. Se hicieron observaciones fenológicas para conocer las épocas de fructificación y, de este modo, obtener semillas para establecer viveros. En éstos se observó el crecimiento de las plantas para determinar la biomasa aprovechable.

Villalobos (1995a, 1996) estudió la distribución de $Q$. amara en Costa Rica en relación con factores ambientales (formación vegetal, iluminación, temperatura, topografía, tipo de suelo, etc.) y contenido de cuasinoides, con la finalidad de dilucidar los requisitos ecológicos de la especie. Él determinó que el factor más importante que condiciona la distribución natural de la especie es la altitud, ya que no existen poblaciones por encima de $500 \mathrm{~m}$. El segundo factor es la gran incapacidad de la especie para tolerar épocas prolongadas de sequía. En regiones de bosque tropical seco sobrevive bajo el bosque de galería, siempre que no haya un déficit hídrico severo.

Las primeras experiencias no sistemáticas de cultivo en Costa Rica datan de 1984 y 1985, en el marco del Proyecto Cooperativo Universidad de Costa Rica (UCR) e Instituto de Desarrollo Agrario (IDA). En 1996 se establecieron parcelas de cultivo sistematizadas en Talamanca, como parte del Proyecto Olafo. A partir de 2000 se estableció una parcela experimental en el Jardín Agroecológico Bougainvillea, con dos modalidades: a) Plantas expuestas al sol y b) Plantas a la sombra de árboles de cacao (Ocampo \& Villalobos 2000). Actualmente se cuenta con una población de 65 mil plantas establecidas en un sistema agroecológico con sombra natural.

\subsection{Domesticación}

La domesticación de recursos naturales nativos es fundamental para lograr el desarrollo agroindustrial, que confiere un valor agregado a la materia prima (Díaz et al. 2004). La puesta en marcha de este proceso contribuye de diversas formas a valorar la diversidad vegetal útil, de modo que se logra:

1. Disminuir la recolección de plantas silvestres, que es el mayor flagelo que propicia la pérdida de recursos naturales en América Latina.

2. Contar con un modelo agroecológico que contribuya en la conservación del ambiente.

3. Contar con materia prima de calidad, obtenida en sistemas de mayor sustentabilidad.

4. Suministrar grandes volúmenes de materia prima para una industrialización adecuada.

5. Demostrar que los sistemas agrícolas tradicionales son importantes sistemas agroecológicos.

Las plantas medicinales y aromáticas, nativas de los bosques neotropicales, tienen potencialmente un gran valor económico; pese a ello, en la región centroamericana existen pocas acciones concretas para impulsar procesos de domesticación que conduzcan a un nuevo enfoque de conservación y desarrollo (Ocampo 1994). Para lograr la conservación y el desarrollo sustentable de los recursos naturales nativos, existen dos alternativas viables: a) Manejo de poblaciones naturales y b) Domesticación.

Villalobos et al. (1997) describen la experiencia del CATIE en el desarrollo de criterios silviculturales para aprovechar de modo sostenible las poblaciones naturales de Quassia amara en un sistema diversificado de manejo del bosque tropical. En el proceso de domesticación es importante considerar las diferentes etapas que constituyen el desarrollo, para contar con alternativas de cultivo viables y diferenciarlas de la clásica domesticación de monocultivo, propia de la agricultura convencional.

La planta medicinal Psychotria ipecacuanha (Brot.) Stokes (Rubiaceae), denominada raicilla en Costa Rica, es la única especie comercial de mercado internacional desde hace seis décadas. En este caso, el sistema de producción agroecológica tradicional es un ejemplo de conservación y de desarrollo de un proceso de domesticación, que favorece la conservación de los bosques naturales (Ocampo 2000, 2004).

Actualmente existe una preocupación creciente por el estado natural de las plantas medicinales, como parte de la biodiversidad. El deterioro del ambiente, causado por la deforestación debida a la presión demográfica, que convierte áreas boscosas en cultivos agrícolas y pastizales para ganado, el uso indiscriminado de productos agroquímicos, así como la contaminación general del aire y del agua, podrán causar la desaparición de muchas especies, incluso antes de haber sido estudiadas. La constante pérdida de biodiversidad en países de América Central y del Caribe afecta directamente la diversidad de plantas medicinales y los ecosistemas donde habitan. Además, las actividades de recolección de plantas silvestres podrían constituir uno de los principales factores causantes de la extinción de algunas especies propias de ambientes boscosos (Ocampo 1994, GermosénGobineau 2005).

Para domesticar plantas medicinales nativas, en el marco de un modelo agroecológico, debe considerarse la estructura biológica de las especies y 
los requerimientos de luz y nutrientes, entre otros, para lograr un manejo adecuado. Al mismo tiempo, en un sistema de producción agroecológica se promueve la conservación in situ de los recursos naturales.

\subsection{Explotación y conservación de plantas medicinales}

Existe una gran riqueza de plantas medicinales y aromáticas en el bosque tropical húmedo. En América Central se usan 932 especies, de las cuales el $85 \%$ son nativas (Ocampo \& Robles 2000). Se sabe que ocurre una activa extracción de recursos naturales (ejemplares enteros, partes y derivados de especies) de ambientes silvestres; estos recursos se incorporan al mercado, lo que puede aumentar la vulnerabilidad de las especies en su medio natural (Lange \& Schippmann 1997).

En Costa Rica, un diagnóstico determinó que se usan 406 especies medicinales y que se comercializan 133 especies; de éstas, el $37 \%$ son plantas silvestres (Ocampo et al. 1994). En la actualidad hay una demanda extensa y creciente, a nivel mundial, de los denominados productos herbarios medicinales, fitofármacos o productos naturales, que se inició con mayor intensidad a partir de la última década del siglo XX, sobre todo en Europa y en los Estados Unidos de Norteamérica (Heinrich et al. 2004). Las medicinas derivadas de plantas constituyen una opción terapéutica útil, que a menudo se considera como una práctica terapéutica segura para muchos padecimientos. Este consumo de medicamentos de origen natural ha provocado, también, un aumento del uso de plantas medicinales y de sus productos elaborados en Centroamérica y el Caribe. Por tanto, es urgente realizar esfuerzos sostenidos para elevar el nivel tecnológico y las garantías de calidad de los medicamentos preparados de plantas de la región (Quintanilla-Almagro 2001).

La Farmacopea Vegetal Caribeña, publicada por ENDA-Caribe a través del Programa TRAMIL (Germosén-Gobineau 2005), incluye 99 especies de plantas medicinales; de éstas, $58 \%$ son nativas de América y $42 \%$ son especies introducidas en la cuenca del Caribe desde otras regiones del mundo. Además, $53 \%$ son especies que se cultivan en mayor o menor grado y solamente $35 \%$ son especies silvestres que, según la parte de la planta cosechada y la intensidad del uso, podrían ser vulnerables a la sobreexplotación. El $11 \%$ de las plantas es una mezcla de poblaciones silvestres y estados incipientes de cultivo (Ocampo \& Robles 2005). Se deduce que aproximadamente $12 \%$ de las especies son vulnerables.

\section{B. PARTE EXPERIMENTAL}

\subsection{Ubicación del área de trabajo}

El Jardín Agroecológico Bougainvillea posee 50 hectáreas de terreno en el área de amortiguamiento de la Reserva de la Biosfera La Amistad Costa Rica-Panamá. Esta área pertenece a la zona de vida bosque tropical muy húmedo, según la clasificación de Holdridge (1987), caracterizada por una alta precipitación la mayor parte del año (precipitación promedio anual de 4000 a $6000 \mathrm{~mm}$ ) y una temperatura media de $25{ }^{\circ} \mathrm{C}$, con una altitud entre 50 y $180 \mathrm{~m}$. El bosque es perennifolio, con pocas especies deciduas, el dosel es alto y abundan las epífitas y los bejucos. El jardín constituye una unidad de producción, que está distribuida de la siguiente manera: $50 \%$ de bosque secundario, $40 \%$ de sistemas agroecológicos y $10 \%$ de áreas para cultivo de plantas medicinales y aromáticas, que requieren de mayor luminosidad.

\subsection{Cultivo agroecológico}

\subsubsection{Manejo de semillas para reproducción}

\subsection{1a. Cosecha de semillas}

En Costa Rica, la floración de Quassia amara tiene lugar entre octubre y abril; los frutos maduran dos meses después. Sin embargo, existe un pico de maduración de frutos entre finales de febrero e inicios de marzo. En el período de maduración, los drupéolos pasan por diversas coloraciones; primero son rojizos, luego se tornan verdes hasta alcanzar un color negro. Los mejores resultados de germinación se han obtenido con drupéolos parcial hasta totalmente negros.

Es importante considerar el brillo y el tamaño de los frutos, ya que éstos son indicadores de la viabilidad de la semilla. Por lo general, frutos con un tamaño entre 1 y $1,5 \mathrm{~cm}$ son adecuados para la reproducción; además, se desprenden fácilmente del receptáculo. En poblaciones naturales es común encontrar infrutescencias secas que permanecen adheridas al árbol; en este caso los drupéolos son opacos, no se desprenden fácilmente del receptáculo y contienen, generalmente, semillas no viables.

En los períodos de fructificación, las poblaciones de $Q$. amara son visitadas por varias especies de aves e insectos, que depredan y dispersan las semillas; esto obliga a realizar cosechas periódicas para obtener suficiente material de calidad.

\subsection{1b. Almacenamiento de semillas}

Las semillas deben contar con un adecuado manejo en el transporte y el almacenamiento, ya que las 
altas temperaturas y la humedad pueden provocar fermentación y reducir la viabilidad. Por tanto, el transporte de semillas debe realizarse en sitios frescos y de fácil ventilación. Los períodos largos de almacenamiento (más de una semana) favorecen la proliferación de hongos y la disminución del poder germinativo. Por esta razón, el despulpado es importante como medida para el manejo adecuado de las semillas; se puede realizar manualmente, frotando las semillas entre las manos, hasta que la pulpa amarillenta se desprenda totalmente.

\subsection{1c. Siembra en vivero}

La germinación de semillas de Quassia amara ocurre en un lapso de 10 semanas; la máxima germinación se observa en la séptima semana y concluye entre la novena y la décima semana. E1 porcentaje de germinación puede variar según el modo de manipular las semillas y el tipo de sustrato utilizado (aserrín, arena, suelo, etc.). A continuación se enumeran varios factores que han permitido mejorar la germinación desde un $50 \%$ hasta un $68 \%$.

1. Control de la sombra. La deshidratación de semillas por insolación disminuye su capacidad de germinación, por lo que se debe controlar la radiación solar en la etapa de vivero. Un $50 \%$ de sombra resultó positivo para la germinación.

2. Riego y dRenaje. Como se anotó anteriormente, la humedad juega un papel importante en la fase de vivero; por tanto, el drenaje de las eras debe ser bueno y debe realizarse un riego diario.

3. Preparación de los bancales o eras. Esto es de particular importancia para el adecuado desarrollo del sistema radicular de las plantas. La remoción del suelo en los primeros $30 \mathrm{~cm}$ mejoró, en general, el desarrollo de las plantas.

4. Densidad de siembra. Una distancia de $10 \times 15 \mathrm{~cm}$, para una densidad de 35 plantas por $\mathrm{m}^{2}$, que puede variar según el tiempo que permanecen las plantas en vivero; se recomiendan no menos de ocho meses. Las semillas se colocan en la superficie, parcialmente cubiertas por aserrín de madera.

5. Control de plagas y enfermedades. Se han encontrado insectos cortadores en los primeros estadios de las plantas, pero no han alcanzado un ataque crítico. También se ha detectado antracnosis, causada por el hongo Colletotrichum sp., que provoca lesiones foliares de color café, rodeadas por un halo amarillento, defoliación y anulación del crecimiento de las plantas. El exceso de humedad por inadecuado control de malezas, mal drenaje del suelo o alta densidad de siembra es un factor que favorece la aparición de esta enfermedad. Es posible controlarla con un fungicida específico.

6. Trasplante. Se realiza ocho meses después de la siembra, cuando las plantas han alcanzado una altura promedio de $40 \mathrm{~cm}$. Se debe utilizar una herramienta apropiada para remover el suelo de las plantas y extraerlas fácilmente sin dañar las raíces, ya que se llevarán con raíces desnudas o en escoba. Además, las plantas deben podarse en los primeros $15 \mathrm{~cm}$ y defoliarse totalmente, para evitar un exceso de deshidratación. Un trasplante inadecuado provocó un ataque de bacterias, que causó una mortalidad de hasta $40 \%$ de plantas en el campo. Por tal razón, se recomienda la protección del material para trasplante con un bactericida.

\subsection{Reproducción asexual}

La metodología empleada es la práctica del acodo aéreo. En general, los ensayos realizados han mostrado que el material juvenil, poco leñoso, difícilmente puede reproducirse asexualmente. Las estacas, tanto juveniles como leñosas, muestran muy bajos rendimientos de prendimiento o desarrollan sistemas radicales muy débiles, que son poco prácticos para la reproducción a gran escala. Los acodos aéreos juveniles (ramas con diámetro menor a $0,9 \mathrm{~cm}$ ) también muestran un prendimiento bajo (10\%); pero en ramas mayores $(1,0$ a 1,5 cm de diámetro) el prendimiento fue muy exitoso (hasta $98 \%$ ). Sin embargo, el establecimiento directo en el campo arrojó resultados negativos (hasta un $50 \%$ de mortalidad). Con el establecimiento en bancal o era, la mortalidad fue de $10 \%$.

\subsection{Establecimiento}

El sistema de cultivo requiere de un adecuado manejo de la sombra, que determina en gran medida un crecimiento óptimo y una materia prima de calidad (con una concentración apropiada de cuasinoides). Los sistemas agroecológicos ofrecen una sombra parcial que, en determinadas condiciones, es ideal para el desarrollo de Quassia amara como cultivo. Un ejemplo son los cacaotales arbolados (Theobroma cacao y Cordia alliodora, principalmente) utilizados como sistema para la producción de cuasia; aquí se podan los árboles de cacao, pero se mantiene el estrato superior de árboles para producción forestal. Asimismo, se realiza limpieza para eliminar la competencia por nutrientes o luminosidad $\mathrm{y}$, de este modo, obtener 
mejor crecimiento y mayor rendimiento.

Recientemente, en un sistema de cacaotal arbolado del Caribe de Costa Rica se midieron aumentos de altura total entre $20 \mathrm{~cm} /$ planta/año y $60 \mathrm{~cm} /$ planta/año en los dos primeros años de la plantación. El aumento de diámetro fue de $0,15 \mathrm{~cm} /$ planta/año a $0,51 \mathrm{~cm} /$ planta/año en los primeros dos años de plantación $(\mathrm{n}=212)$. Por otra parte, a partir de plántulas obtenidos de semillas, con distanciamientos de $1,2 \times 1,2 \mathrm{~m}$, con sombra parcial y a plena luz, el cuarto año se obtuvo una biomasa aprovechable de $0,7 \mathrm{~kg}$, con un incremento promedio de $0,168 \mathrm{~kg} /$ planta/año $(\mathrm{n}=25)$. El rendimiento en condiciones de cultivo agroecológico, con 2500 plantas por hectárea, fue de $2250 \mathrm{~kg}$ de madera fresca. En ensayos posteriores se espera obtener más cosechas en menor tiempo y aumentar los rendimientos de materia prima con un contenido óptimo de cuasinoides.

Estos criterios de plantación se deben, en primera instancia, a las condiciones del hábitat, que se mencionaron anteriormente, y promueven el desarrollo del cultivo en un sistema que ha sido considerado como poco productivo. No obstante, es necesario indicar que la cuasia es sólo uno de los componentes entre las opciones de un sistema de cultivo agroecológico.

\subsection{Manejo de rebrotes}

Una cualidad ventajosa en el manejo de la biomasa aprovechable de Quassia amara es su capacidad para rebrotar. Esta cualidad está determinada por la edad de la planta, reflejada en el grosor del tallo del que se originan los rebrotes, y por el grado de iluminación donde se halla esa planta. El crecimiento óptimo de estos rebrotes, tanto en poblaciones naturales como en plantaciones, se da en función de los siguientes elementos:

1- Altura de corte para cosecha. El primer corte para cosecha se realiza entre 30 y $50 \mathrm{~cm}$ de altura desde el nivel del suelo. Esto garantiza un número adecuado de rebrotes productivos para la próxima cosecha, sin poner en riesgo la planta.

2- Dí́metro para la cosecha. La cosecha óptima se realiza cuando los tallos tienen un grosor de 2 a $2,5 \mathrm{~cm}$, lo que permite un adecuado crecimiento.

3- TIPO DE CORTE. El corte se realiza en forma transversal-inclinada, sin provocar daños en el tallo remanente; esta medida evita el exceso de humedad en el corte, disminuyendo el riesgo de infección.

4- Poda de ReBrotes. En evaluaciones preliminares, la poda de los rebrotes de menor desarrollo estimula el crecimiento de los rebrotes productivos; sin embargo, se ha observado que los rebrotes menores sufren autopoda; es decir, se eliminan naturalmente.

5- Mantenimiento de la sombra. Para lograr un crecimiento adecuado de los rebrotes, debe haber sombra apropiada; la alta luminosidad inhibe el crecimiento, al igual que el exceso de sombra.

\subsection{Rendimiento de rebrotes}

Para el experimento se utilizaron plantas de cinco años de edad, con altura de corte de $0,3 \mathrm{~m}$ y diámetros en la base de 2,0 a 3,0 cm. Se efectuó una primera evaluación de los rebrotes aparecidos luego de un período de un año. Como resultado se obtuvo un promedio de tres rebrotes por planta. Estos rebrotes tuvieron alturas comprendidas entre 1,7 y $2,2 \mathrm{~m}$ y diámetros entre 0,9 y $1,7 \mathrm{~cm}$.

\section{Fitoquímica y domesticación}

El manejo adecuado de poblaciones naturales y la domesticación de plantas nativas con acción biológica (medicinal, insecticida, herbicida, etc.) tienen que cumplir con un criterio de calidad, que en este caso es un contenido aceptable de metabolitos secundarios. Además, tienen que contribuir con varios componentes importantes:

1. Social; puede beneficiar económicamente a poblaciones marginadas.

2. ECOLÓGICO; contribuye con la conservación de bosques por el aprovechamiento racional y sostenible de una o más especies de plantas.

3. INDUSTRIAL; podrán establecerse pequeñas o medianas empresas para procesar materias primas valiosas.

4. SALUD; diversos sectores de la sociedad podrán aprovechar mejor las virtudes medicinales de las plantas, porque habrá material suficiente y homogéneo con control de calidad.

Los estudios fitoquímicos son fundamentales para controlar los principios activos, que son la base de la acción biológica de las plantas. Es necesario comparar la composición química, tanto cualitativa como cuantitativa, del material obtenido en diversos ambientes. La domesticación será exitosa si en el ambiente de cultivo se biosintetizan los principios activos en cantidad suficiente, de modo que se pueda determinar el potencial productivo y las posibilidades futuras de comercialización, para proponer posteriormente un método de industrialización económicamente viable.

\subsection{Aislamiento de cuasina y neocuasina}

En la literatura se encuentran varios métodos para extraer, separar y purificar los constituyentes de los extractos de cuasia (Mora 1995). Para la identificación 
de los compuestos principales (utilizados como marcadores) de las muestras de madera de Quassia amara, se realizó extracción, separación y purificación de cuasina y neocuasina principalmente con métodos cromatográficos (Fig. 1). Estos compuestos se identificaron con métodos espectroscópicos (resonancia magnética nuclear, infrarrojo, masas, etc.) y se usaron como compuestos patrón para efectuar análisis cuantitativos de extractos alcohólicos de materiales de diversos grosores y, por tanto, para realizar un control de calidad de los productos medicinales obtenidos.

6.2. Análisis de muestras de madera de distintos grosores

\subsubsection{Muestras cultivadas de cuasia}

La madera (troncos y ramas) proveniente de los cultivos experimentales del Jardín Agroecológico Bougainvillea, establecidos en dos ambientes diferentes, con una edad de 5 años, se secó hasta peso constante y se molió. Las muestras eran troncos de aproximadamente $2 \mathrm{~cm}$ de diámetro (A), ramas de aproximadamente $1 \mathrm{~cm}$ de diámetro $(\mathrm{H})$ y ramas de un grosor menor a $1 \mathrm{~cm}$ de diámetro $(\mathrm{N})$. Con el material seco y molido se hicieron extracciones con etanol al $95 \%$, que se analizaron según la metodología descrita por Mora (1995). Los análisis cuantitativos se realizaron mediante cromatografía líquida de alta eficiencia (HPLC), utilizando como patrones los dos compuestos purificados (Mora 1995).

\subsubsection{Resultados en madera de distintos grosores}

La Fig. 6 muestra las cifras del contenido de cuasina en distintos ejemplares cultivados de Quassia amara. Cada barra de la figura representa el promedio de nueve mediciones. La Fig. 7 muestra las cifras del contenido de neocuasina. Como se observa en estas dos figuras, la concentración de los compuestos aumenta con el grosor del tallo o de las ramas; en otras palabras, aumenta con la edad. A partir de ejemplares sembrados a la sombra se obtuvo mayor cantidad de cuasina, mientras que la cantidad de neocuasina es levemente superior en muestras procedentes de áreas expuestas al sol.

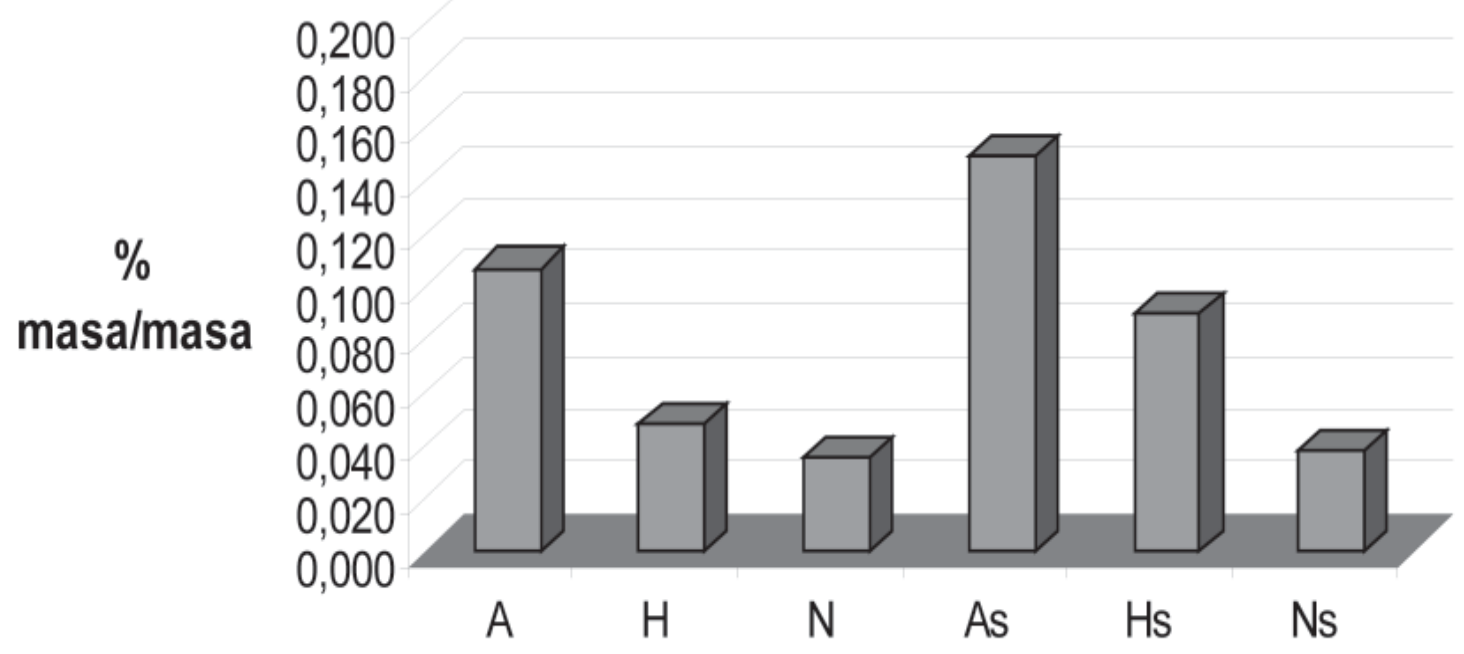

A, $\mathrm{H}$ y $\mathrm{N}=$ plantas cultivadas y expuestas al sol; As, Hs y Ns = plantas cultivadas con sombra

As y $\mathrm{A}=$ troncos de aproximadamente $2 \mathrm{~cm}$ de diámetro

Hs y $\mathrm{H}=$ ramas de aproximadamente $1 \mathrm{~cm}$ de diámetro

Ns y $\mathrm{N}=$ ramas de menos de $1 \mathrm{~cm}$ de diámetro

Fig. 6. Contenido de cuasina en muestras cultivadas de Quassia amara. 


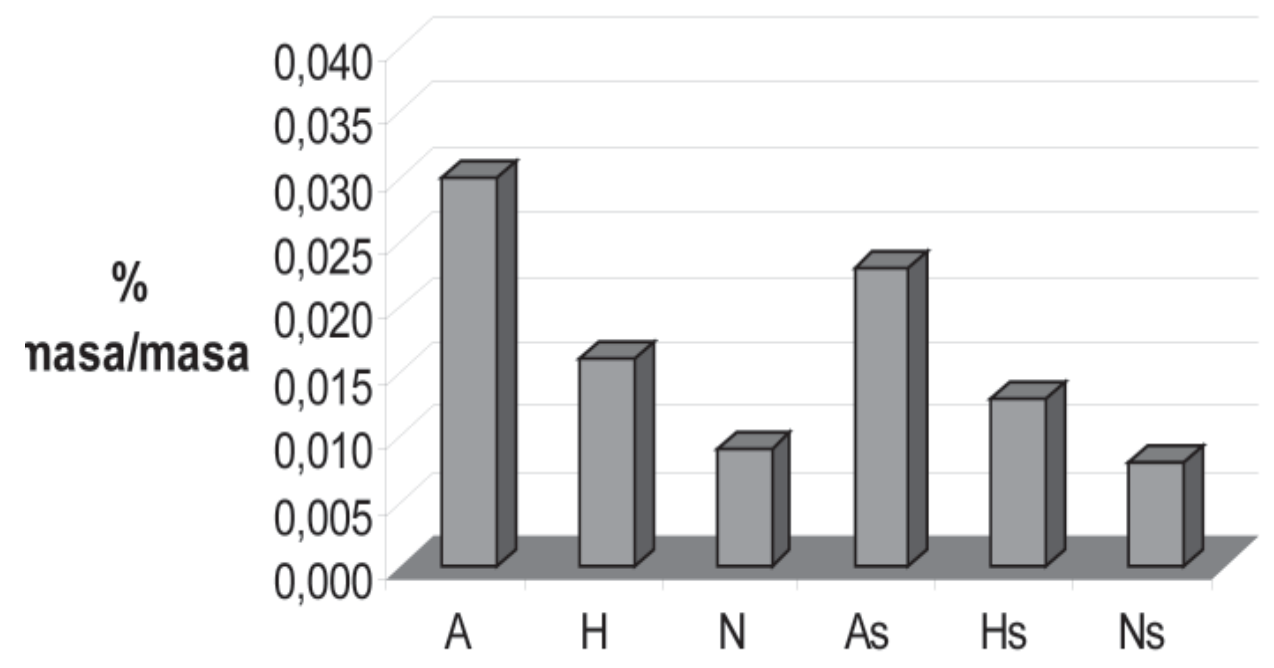

A, $\mathrm{H}$ y $\mathrm{N}$ = plantas cultivadas y expuestas al sol; As, Hs y $\mathrm{Ns}=$ plantas cultivadas con sombra As y $\mathrm{A}=$ troncos de aproximadamente $2 \mathrm{~cm}$ de diámetro $\mathrm{Hs} \mathrm{y} \mathrm{H}=$ ramas de aproximadamente $1 \mathrm{~cm}$ de diámetro Ns y $\mathrm{N}=$ ramas de menos de $1 \mathrm{~cm}$ de diámetro

Fig. 7. Contenido de neocuasina en muestras cultivadas de Quassia amara.

\section{Conclusiones}

a) Las plantas cultivadas siguen el patrón observado en muestras de la especie obtenidas del medio natural (Villalobos 1995b, Villalobos et al. 1999); es decir, a mayor edad de la planta, mayor es la acumulación de cuasinoides en el xilema.

b) Las condiciones de cultivo, a la sombra o al sol, influyen notablemente en la cantidad de cuasina acumulada por las plantas. Este factor tendrá que tomarse en cuenta para lograr una domesticación tecnificada.

c) Las plantas procedentes de bosques tropicales, que se incorporan a procesos de domesticación, deben ser evaluadas a lo largo del tiempo mediante estudios fitoquímicos sistemáticos, para determinar el momento de cosecha adecuado.

d) Los primeros resultados del modelo de cultivo de Quassia amara, que fue evaluado, evidencian la adaptación de esta especie a un sistema agroecológico. Con las condiciones de luminosidad controladas, este sistema favorece tanto el crecimiento de las plantas, como la concentración de cuasinoides en los tejidos, lo que aumenta la calidad de la materia prima.

Agradecimientos. Agradecemos al proyecto regional
"Desarrollo de Tecnología de Cultivo y Producción de Fitoterápicos" (Proy. OEA/AICD/USAC/No. AE/089/01) por su apoyo económico, a J.C. Brenes (CIPRONA) por su ayuda en las labores de laboratorio, a N.R. Farnsworth (College of Pharmacy, University of Illinois, Chicago) por su ayuda para consultar la base de datos NAPRALERT, y a los revisores del artículo por sus valiosas correcciones y sugerencias.

\section{LITERATURA CITADA}

Abdel-Malek, S., J.W. Bastien, W.F. Mahler, Q. Jia, M.G. Reinecke, W.E. Robinson Jr., Y.H. Shu \& J. Zalles-Asin. 1996. Drug Leads from the Kallawaya Herbalists of Bolivia. 1. Background, rationale, protocol and anti-HIV activity. J. Ethnopharmacol. 50(3): 157-166.

Ajaiyeoba, E.O., U.I. Abalogu, H.C. Krebs \& A.M.J. Oduela. 1999. In vivo antimalarial activities of Quassia amara and Quassia undulata plant extracts in mice. J. Ethnopharmacol. 67(3): 321-325.

Badilla, B., T. Miranda, G. Mora \& K. Vargas. 1998. Actividad gastrointestinal del extracto acuoso bruto de Quassia amara (Simarubaceae). Rev. Biol. Trop. 46(2): 203-210.

Barbetti, P., G. Grandolini, G. Fardella \& I. Chiappini. 1987. Indole alkaloids from Quassia amara. Planta 
Med. 53(3): 289-290.

Barbetti, P., G. Grandolini, G. Fardella, I. Chiappini \& A. Mastalia. 1990. New canthin-6-one alkaloids from Quassia amara. Planta Med. 56(2): 216-217.

Barbetti, P., G. Grandolini, G. Fardella \& I. Chiappini. 1993. Quassinoids from Quassia amara. Phytochemistry 32(4): 1007-1013.

Cáceres, A., T. Mejía, R.A. Ocampo \& R. Villalobos. 1995a. Quassia amara L. ex Blom (Simaroubaceae). Revisión bibliográfica. In: Ocampo, R.A. (ed.). Potencial de Quassia amara como insecticida natural. Informe Técnico No. 267. CATIE, Turrialba, Costa Rica [Otras referencias citadas de ese trabajo]. p. 159-178.

Cáceres, A., E. Jáuregui \& R. Villalobos. 1995b. Efecto de las condiciones ambientales de crecimiento en la actividad antimicrobiana de Quassia amara. In: Ocampo, R.A. (ed.). Potencial de Quassia amara como insecticida natural. Informe Técnico No. 267. CATIE, Turrialba, Costa Rica. p. 97-99.

Cañigueral, S., R. Vila \& M. Wichtl. 1998. Plantas medicinales y drogas vegetales para infusión y tisana. OEMF International, Milán. p. 424-425.

Cecchini, T. 1971. Enciclopedia de las hierbas y de las plantas medicinales. De Vecchi, Barcelona. p. 153.

Centro Nacional de Acción Pastoral. 1991. Recetas y razones para una salud natural. CENAP, San José, Costa Rica. p. 87.

Claus, E.P. \& V.E. Tyler. 1965. Farmacognosia. E1 Ateneo, Buenos Aires. p. 133.

Cronquist, A. 1944. Studies in the Simaroubaceae - IV Resume of the American genera. Brittonia 5: 128147.

Cubillo, D., G. Sanabria \& L. Hilje. 1997. Mortalidad de adultos de Bemisia tabaci con extractos de hombre grande (Quassia amara). Manejo Integrado de Plagas (Costa Rica) 45: 25-29.

Díaz, R., J.F. Cicció \& R.A. Ocampo. 2004. Domesticación de recursos naturales nativos en condiciones agroecológicas en el trópico húmedo en el Caribe de Costa Rica. In: Canuto, J.C. \& J.A. Costabeber (eds.). Agroecologia. Conquistando a Soberania Alimentar, Embrapa Clima Temperado, Pelotas, RS, Brasil. p. 193-212.

Evans, D.A. \& R.K. Raj. 1988. Extracts of Indian plants as mosquito larvicides. Indian J. Med. Res. 88(1): 38-41.

Fernand, V.E. 2003. Initial Characterization of Crude Extracts from Phyllanthus amarus Schum. and Thonn. and Quassia amara L. using Normal Phase Thin Layer Chromatography. Master Thesis, Louisiana St. Univ. 70 p.
Fernando, E.S. \& C.J. Quinn. 1995. Picramniaceae, a new family, and a recircumscription of Simaroubaceae. Taxon 44: 177-181.

Ferrigni, N.R., J.E. Putnam, B. Anderson, L.B. Jacobsen, D.E. Nichols, D.S. Moore, J.L. Mc Laughlin, R.G. Powell \& C.R. Smith Jr. 1982. Modification and evaluation of the potato disc assay and antitumor screening of Euphorbiaceae seeds. J. Nat. Prod. 45(6): 679-686.

Fournier, L.A. \& E.G. García. 1998. Nombres vernaculares y científicos de los árboles de Costa Rica. San José, Ed. Guayacán. p. 75.

Furlan, R., G. Molina, O. Micheloni, M. Zudenigo \& S. Zacchino. 1994. Uso del bioensayo de Artemia salina para la detección de productos naturales con actividad biológica de la familia Simaroubaceae. Anales de S.A.I.P.A. (Buenos Aires) 12: 293-312.

García, H. 1992. Flora medicinal de Colombia. Botánica Médica II. $2^{\mathrm{a}}$ ed. Bogotá, Tercer Mundo. p. 43-47.

García, M., S.M. González \& L. Pazos. 1996-1997. Actividad farmacológica del extracto acuoso de la madera de Quassia amara (Simaroubaceae) en ratas y ratones albinos. Rev. Biol. Trop. 44(3) / 45(1): 4750 .

Germosén-Gobineau, L. (ed.). 2005. Farmacopea Vegetal Caribeña. 2a ${ }^{\mathrm{a}}$. ed. TRAMIL, León, Edit. Universitaria, UNAN. 486 p.

Heinrich, M., J. Barnes, S. Gibbons \& E.M. Williamson. 2004. Fundamentals of Pharmacognosy and Phytotherapy. London, Churchill Livingstone. $320 \mathrm{p}$.

Holdridge, L.R. 1987. Ecología basada en zonas de vida. $3^{\mathrm{a}}$ reimpresión. San José, IICA. 216 p.

Holdridge, L.R. \& L.J. Poveda. 1975. Árboles de Costa Rica. Vol. I. San José, Centro Científico Tropical. $546 \mathrm{p}$.

Holdridge, L.R., L.J. Poveda \& Q. Jiménez. 1997. Árboles de Costa Rica. Vol. I, $2^{a}$ ed. San José, Centro Científico Tropical. p. 412.

Kupchan, S.M. \& D.R. Streelman. 1976. Quassimarin, a new anti-leukemic quassinoid from Quassia amara. J. Org. Chem. 41: 3431.

Lange, D. \& U. Schippmann. 1997. Trade Survey of Medicinal Plants in Germany. A Contribution to International Plant Species Conservation. Bonn, Bundesamt für Naturschutz. 128 p.

Ling, F. 1995. Estudio ecológico de Quassia amara en la Reserva Indígena de Kéköldi, Costa Rica. In: Ocampo, R.A. (ed.). Potencial de Quassia amara como insecticida natural. Informe Técnico No. 267. CATIE, Turrialba, Costa Rica. p. 56-67. 
Marmillod, D., Y. Chang \& R. Bedoya. 1995. Plan de aprovechamiento sostenible de Quassia amara en la Reserva Indígena de Kéköld. In: Ocampo, R.A. (ed.). Potencial de Quassia amara como insecticida natural. Informe Técnico No. 267. CATIE, Turrialba, Costa Rica. p. 68-90.

Manfred, L. 1947. 7.000 recetas botánicas a base de 1.300 plantas medicinales americanas. Kier, Buenos Aires. 778 p.

Mora, G.A. 1995. Extracción y estudio cromatográfico de extractos de Quassia amara. In: Ocampo, R.A. (ed.). Potencial de Quassia amara como insecticida natural. Informe Técnico No. 267. CATIE, Turrialba, Costa Rica. p. 93-96.

Morton, J.F. 1981. Atlas of Medicinal Plants of Middle America. Bahamas to Yucatan. Springfield, IL., C.C. Thomas. p. 389-390.

Ninci, M.E. 1991. Profilaxis y tratamiento de pediculosis con cuasia amarga. Rev. Fac. Cienc. Med. Univ. Nac. Córdoba 49(2): 27-31.

Njar, V.C.O., T.O. Alao, J.I. Okagun \& H.L. Holland. 1993. 2-methoxycanthin-6-one: a new alkaloid from the stem wood of Quassia amara. Planta Med. 59(3): 259-261.

Núñez-Meléndez, E. 1975. Plantas medicinales de Costa Rica y su folclore. San José, Edit. Univ. Costa Rica. p. 213.

Ocampo, R.A. 2000. Agrotecnología para el cultivo de ipecacuana o raicilla. In: Martínez, J.V., H.Y. Bernal \& A. Cáceres (eds.). Fundamentos de agrotecnología de cultivo de plantas medicinales iberoamericanas. Santafé de Bogotá, CAB-CYTED. p. 357-374.

Ocampo, R.A. 2004. Ipecacuana, Psychotria ipecacuanha (Brotero) Stokes: Un producto no maderable cultivado bajo el bosque en Huetar Norte, Costa Rica. In: Alexiades, M.N. \& P. Shanley (eds.). Productos Forestales, Medios de Subsistencia y Conservación. Vol. 3 - América Latina. Estudios de caso sobre sistemas de manejo de productos forestales no maderables. CIFOR, SMK Desa Putera, Jakarta, Indonesia. p. 257-273.

Ocampo, R.A. 1994. La domesticación de plantas medicinales. Antecedentes de la reunión. In: Ocampo, R.A. (ed.). Domesticación de plantas medicinales en Centroamérica. Informe técnico No. 245. CATIE, Turrialba, Costa Rica. p. 5-10.

Ocampo, R.A., T. Palma \& N. Hidalgo. 1994. Diagnóstico de Costa Rica. In: Ocampo, R.A. (ed.). Domesticación de plantas medicinales en Centroamérica. Informe técnico No. 245. CATIE, Turrialba, Costa Rica. p. 50-66.

Ocampo, R.A. \& X. Robles. 2000. Índice de Plantas
Medicinales de Centro América. (Mimeogr.)

Ocampo, R.A. \& R. Villalobos. 2000. Agrotecnología para el cultivo de hombre grande o cuasia. In: Martínez, J.V., H.Y. Bernal \& A. Cáceres (eds.). Fundamentos de agrotecnología de cultivo de plantas medicinales iberoamericanas. Santafé de Bogotá, CAB-CYTED. p. 375-386.

Ocampo, R. \& X. Robles. 2005. Estado de conservación de las plantas medicinales TRAMIL: un aporte a la implementación de la estrategia global para la conservación vegetal. San José, Lara Segura \& Asoc. $72 \mathrm{p}$.

Parveen, S., S. Das, C.P. Kundra \& B.M.J. Pereira. 2003. Acomprehensive evaluation of the reproductive toxicity of Quassia amara in male rats. Reprod. Toxicol. 17: 45-50.

Pittier, H. 1957. Ensayo sobre plantas usuales de Costa Rica. $2^{a}$ ed. San José, Univ. Costa Rica. 264 p.

Poveda, L.J. 1995. Taxonomía de Quassia amara y su distribución en el Neotrópico. In: Ocampo, R.A. (ed.). Potencial de Quassia amara como insecticida natural. Informe Técnico No. 267. CATIE, Turrialba, Costa Rica. p. 11-13.

Quintanilla A., E. 2001. ¿Hacia adónde va el mercado de plantas y productos derivados de Iberoamérica? In: Memoria del Seminario Iberoamericano de Armonización de la Legislación y Estado de la Comercialización de Plantas Medicinales y Productos Derivados. Antigua, Guatemala, 29-31 agosto 2001. p. 59-61.

Roark, R.C. 1947. Some promising insecticidal plants. Econ. Bot. 1: 437-445.

Robins, R.J.\& M.J.C. Rhodes. 1984. High-performance liquid chromatographic methods for the analysis and purification of quassinoids from Quassia amara L. J. Chrom. 283: 436-440.

Rodríguez, H. \& F.A. Blanco. 1995. Uso de cuatro extractos orgánicos para el control del pulgón verde. In: Ocampo, R.A. (ed.). Potencial de Quassia amara como insecticida natural. Informe Técnico No. 267. CATIE, Turrialba, Costa Rica. p. 110-114.

Segleau, J. 2001. Plantas medicinales en el trópico húmedo. San José, Ed. Guayacán. p. 102-103.

Simão, S.M., E.L. Barreiros, M.F. Da Silva \& O.R. Gottlieb. 1991. Chemogeographical evolution of quassinoids in Simaroubaceae. Phytochemistry 30: 853-865.

Toma, W., J. de S. Gracioso, F.D.P. de Andrade, C.A. Hiruma-Lima, W. Vilegas \& A.R.M. Souza Brito. 2002. Antiulcerogenic activity of four extracts obtained from the bark wood of Quassia amara L. (Simaroubaceae). Biol. Pharm. Bull. 25: 1151-1155. 
Toma, W., J. de S. Gracioso, C.A. Hiruma-Lima, F.D.P. de Andrade, W. Vilegas \& A.R.M. Souza Brito. 2003. Evaluation of the analgesic and antiedematogenic activities of Quassia amara bark extract. J. Ethnopharmacol. 85: 19-23.

Villalobos, R. 1995a. Distribución natural de Quassia amara en Costa Rica. In: Ocampo, R.A. (ed.). Potencial de Quassia amara como insecticida natural. Informe Técnico No. 267. CATIE, Turrialba, Costa Rica. p. 14-47.

Villalobos, R. 1995b. Distribución de Quassia amara L. ex Blom en Costa Rica, y su relación con los contenidos de cuasina y neocuasina (insecticidas naturales) en sus tejidos. Tesis M. Sc., CATIE, Turrialba, Costa Rica. 174 p.

Villalobos, R. 1996. Caracterización de la distribución de una planta medicinal (Quassia amara) como base para su manejo técnico. X Congreso Agronómico
Nacional. San José. p. 17-22.

Villalobos, R., Y. Chang, D. Marmillod, R. Bedoya \& L. Leigue. 1997. Desarrollo de criterios silviculturales para el manejo de Quassia amara, un producto no maderable del bosque tropical. Simposio Internacional "Posibilidades de Manejo Forestal Sostenible en América Tropical", Santa Cruz de la Sierra, Bolivia. p. 1-6.

Villalobos, R., D. Marmillod, R. Ocampo, G. Mora \& C. Rojas. 1999. Variations in the quassin and neoquassin content in Quassia amara (Simaroubaceae) in Costa Rica. Ecological and management implications. Acta Hort. 502: 369-375.

Zamora, N., Q. Jiménez \& L.J. Poveda. 2000. Árboles de Costa Rica. Vol. 2. Centro Científico Tropical, Conservación Internacional \& Instituto Nacional de Biodiversidad. Santo Domingo de Heredia, Ed. INBio. 374 p. 